Off they go:

\section{Single-use Technology in Future Production BioTech 2016 Conference at ZHAW Waedenswil}

\author{
Elsbeth Heinzelmann, science and technology \\ journalist
}

Abstract: "Keep up with today's single-use technology standard but also remain one step ahead" - that could have been the motto of the BioTech 2016 conference at ZHAW Waedenswil on September 5 and 6, which brought together manufacturers, suppliers, users, regulatory specialists and representatives of organizations in this field to implement single-use devices all over the world. It was an effectiveness analysis of the trend to replace reusable equipment in production facilities with singleuse components.

\section{Keywords: Antibodies · Bioreactor systems/technology ·} Cell \& gene therapy CGT · mAbs/ADCs .

Single-use bioprocess platform

In early September 2016, the Institute of Chemistry and Biotechnology at the Zurich University of Applied Sciences in Waedenswil became the focus for industrial companies who wanted to learn about the state-of-the-art in cell-based $\mathrm{mAb}$ and vaccine production, biosimilars and microbial products, as well as the implementation of single-use technology in the development and manufacture of cell therapeutics. The idea was to create innovative new solutions for international markets. The event was divided into speeches and poster presentations, a postconference workshop and a mini-symposium focusing on plant cells called 'From Nature to Bioreactor'. Let's take a look at some of the selected topics:

Single-Use Technology, run by Regine Eibl, is also one of the Thematic Platforms of NTN Swiss Biotech and biotechnet Switzerland. The platforms bring together scientists from industry and academia dedicated to a shared topic. The ultimate goal is to initiate joint research projects and thereby facilitate innovation.

\section{The Single-use Technologies are Heading for Success}

Right at the start of the event, Eric Langer, President and Managing Partner of BioPlan Associates Inc. in Rockville/ Maryland, showed how future single-use system (SUS) developments will affect the production of therapeutics and biosimilars over the next five years. Today, the global market is estimated to be about US $\$ 200$ billion, with an annual growth rate of around $15 \%$. As single-use manufacturing opens up strong potential for new opportunities, single-use bioprocessing equipment now dominates, accounting for $\geq 85 \%$ of the smaller pre-commercial bioprocessing market. "The market for singleuse equipment will grow rapidly, doubling in five years, as new commercial scale-facilities are projecting they will split certain unit operations about 50:50 between stainless steel and singleuse in five years", prophesies Eric Langer, who has over 20 years' experience in biotechnology and life sciences international marketing, management and market assessment. "As to biosimilars, we expect capacity demands to increase sixfold in the next five years. Although this demand can be met with a mix of production platforms, the trend is towards single-use, as there are several reasons for this: demand for greater cost-containment and control, more productivity and efficiency, manufacture in developing regions, worldwide standardization of biomanufacturing, the use of contract manufacturing organizations, the dominance of single-use bioprocessing equipment for clinical trials-scale manufacturing, especially for smaller and underfunded companies to reduce facilities construction costs among others. For all bioprocessing, new product areas in demand include six of the top seven areas involving single-use devices. Topping the list are downstream continuous bioprocessing technologies.

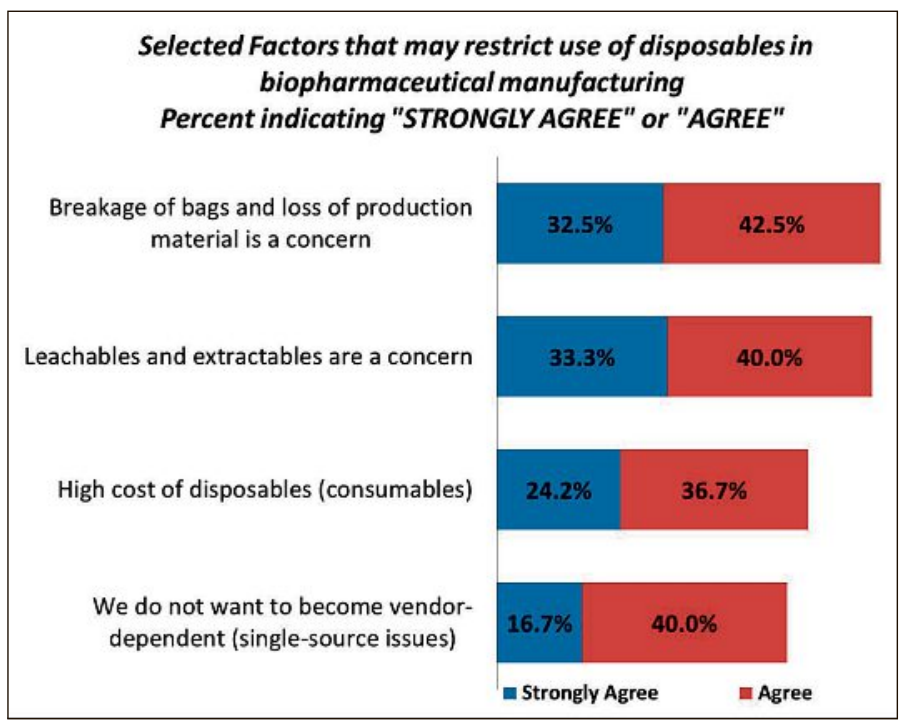

Leachables/Extractables. (Source: $13^{\text {th }}$ annual report and survey of biomanufacturing. www.BioPlan Associates.COM/13th)

"The SUS 'enabling technologies' allow a better, faster, more consistent bioprocessing at ever larger scales", explains Eric Langer, who has held senior management positions at biopharmaceutical supply companies. As he explains, singleuse batch-fed bioreactors are most likely to be recommended by $71.5 \%$ of the industry for new clinical-scale products. The most rapid adoption of SUS devices today includes the most complex devices, such as mixing systems, membrane adsorbers and bioreactors. "There are still a few hard nuts to crack, including problems with breakage and with leachables/extractables data. We suppose that $76 \%$ and $73 \%$, respectively, of the industry may still be holding back on adoption until these issues are resolved."

http://bioplanassociates.com

elanger@bioplanassociates.com 


\section{Could Drugs be more Affordable?}

Miriam Monge, Director of Marketing at Sartorius Stedim Biotech, raised the topic of smart design and implementation of fully single-use bioprocess platforms for mAbs, advanced drug candidates (ADCs) and vaccines. As she recalls, the biotechnology industry has to meet new challenges like the need to increase supply, greater complexity, the demand for greater productivity at a lower cost and increased regulatory oversight. Flexible manufacturing operations enable smaller facilities to be operated around the globe and shorten the time to market, minimizing both capital outlays and cost of goods. "Drug manufacturers can build facilities that utilize single-use technology in 12 months or less compared to the 24 months required for a stainless steel facility", explains the internationally recognized expert from the biopharmaceutical industry. "Plant utilization is higher because of reduced downtime between batches, which operators need for cleaning and sterilizing multi-use equipment. Lower utility requirements enable a 20 to $40 \%$ reduction in facility footprints." A further major advantage is that flexible facilities are greener, generating around half the $\mathrm{CO}_{2}$ emissions of stainless steel plants while also being safer because of reduced risk of product cross contamination.

The operation of flexible facilities requires great assurance of supply from the manufacturers of single-use technologies. Sartorius uses its material science, Quality by Design, film extrusion, bag making and product design expertise to ensure

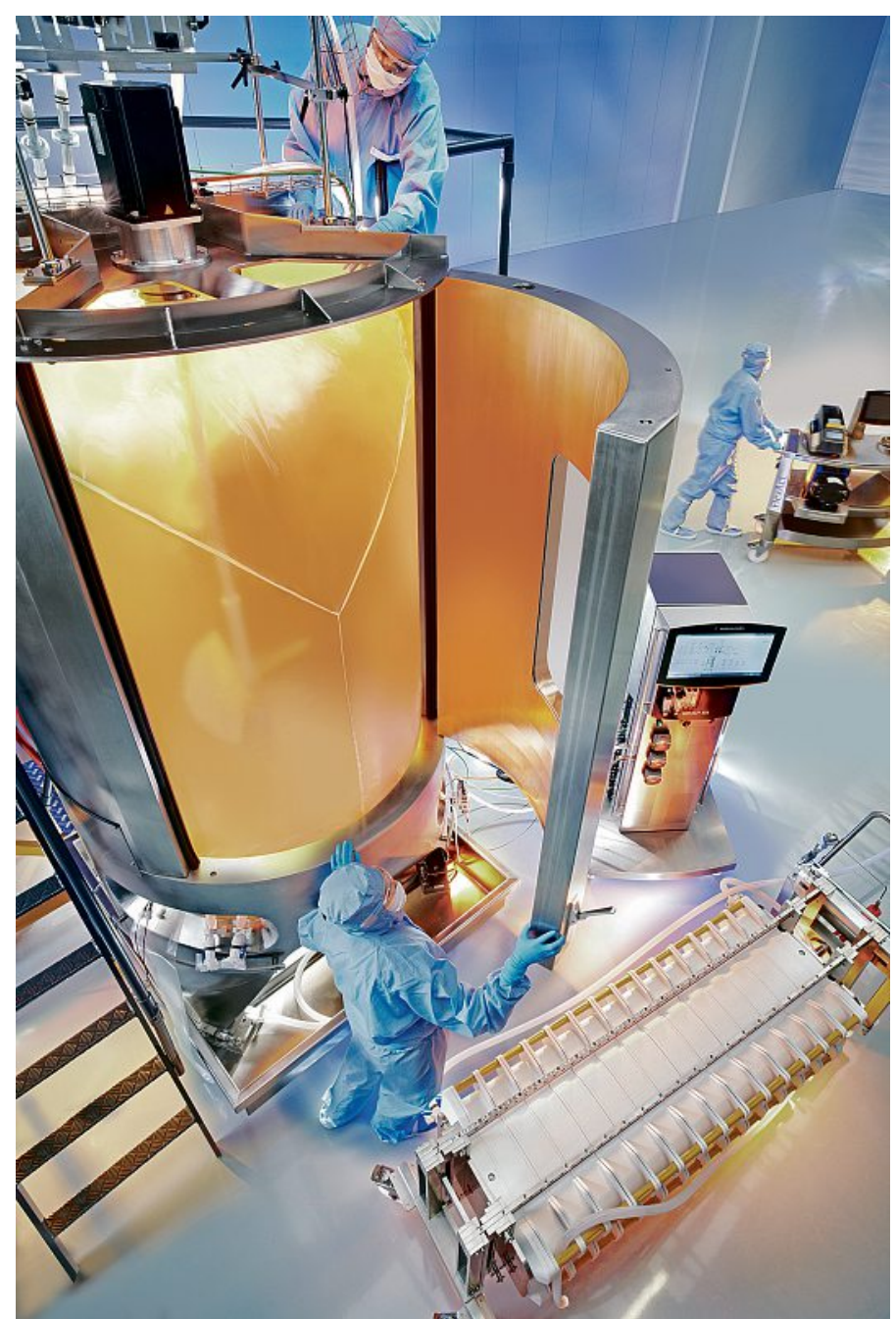

The BIOSTAT ${ }^{\circledR}$ STR of Sartorius offers true scalability in single use and is ideal for high density cell culture applications, monoclonal antibody, recombinant protein and vaccine production as well as seed cultivation for large-scale bioreactors. (Source Sartorius Stedim) it is always able to deliver consumables to customers. The company's assurance of supply policy is built on partnerships, transparency, supply contracts and complete control of the supply chain and manufacturing process, from the resins to the final SU predesigned solution. The industry can benefit from these standardized single-use bioprocess platforms. "At Sartorius we are developing these for molecule classes such as mAbs, ADCs, vaccines and continuous/intensified processing", comments Miriam Monge, who sees her company Sartorius Stedim Biotech as the ideal partner for designing and implementing entire singleuse or hybrid production processes from PD to commercial manufacture. "Based upon our extensive experience with projects for these molecules, our process development consultants can recommend how Sartorius can implement these platforms to meet the specific needs of customers without compromising on the speed or cost benefits associated with this approach."

www.sartorius.com

miriam.monge@sartorius-stedim.com

\section{In the World of Increased Drug Efficacy}

Regarding the novel development in applications with mammalian cells, Klaus Kaiser, Head of Downstream Processing at Bayer Health Care, outlined breakthrough opportunities from the developer's perspective. Antibodies still dominate the field of biopharmaceutical drugs, and many of them fill the pipelines and are waiting to be produced for added patient benefit. "When antibodies are combined with a small molecule, for example a very toxic one, they can be transformed into precise weapons, killing cancer cells very specifically", says the researcher who studied at the Ludwig Maximilian University in Munich. Some of Bayer's lead candidates are such ADCs that target cell surface proteins, which are overexpressed in various very aggressive cancers. "Strong efficacy signals in ongoing clinical trials point to an accelerated development scenario. How can a CMC (Chemistry, Manufacturing \& Controls) function prepare for such a potential breakthrough?" The development programme depends on the complexity of the product, the availability of platform technologies, relevant prior knowledge and the timing of designation. Risk assessments and comparability protocols are key considerations. It is a challenging task to ensure launch readiness on a manufacturing scale against the background of shortened timelines. The clinical manufacturing process and corresponding facilities already need to meet similar quality/ GMP expectations as the subsequent commercial manufacturing.

Usually ADCs are produced in dedicated facilities. "The use of disposable systems and glove box technology enables

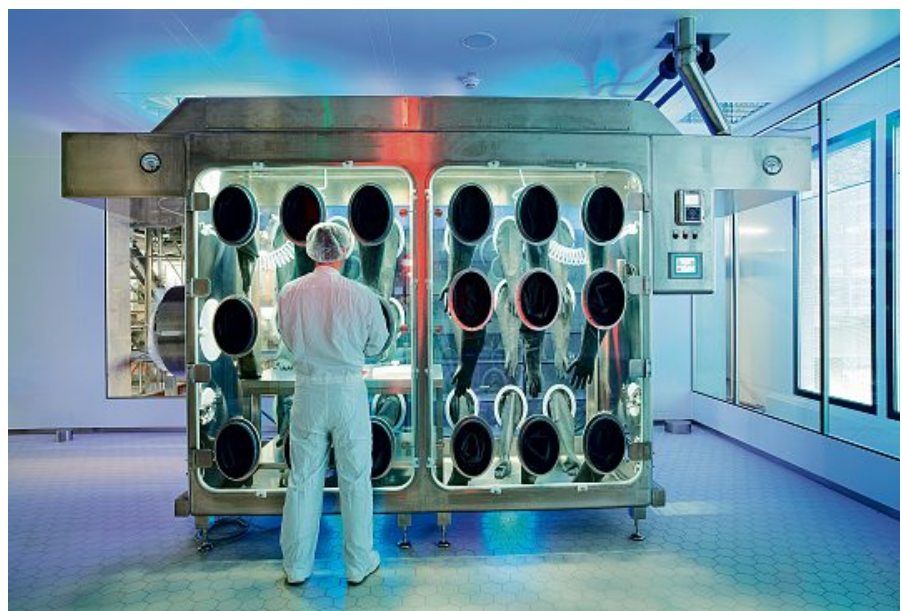

Employee at an isolator for the manufacture of ADCs at Bayer Pharma. (Source Bayer Pharma) 
us to perform clinical manufacturing in multi-purpose facilities where both antibodies and ADCs can be produced in the same cleanroom", concludes Klaus Kaiser, who has been working with ADCs for more than a decade now. Cross contamination is prevented by using dedicated equipment and single-use technologies in a closed system for conjugation and purification. This could support the use of initial product supply from a clinically proven site."

www.pharma.bayer.com

klaus.kaiser@bayer.com

\section{Production of Cell Therapeutics}

On the subject of the implementation of single-use technologies in the development and production of cell and gene therapeutics, Christian van den Bos lays out his views regarding the commercial manufacture of cell therapeutics. Today therapeutic cells are largely manufactured in static systems such as cell factories. "It is widely acknowledged that closed processes are advantageous, but converting processes based on such a technology is fraught with difficulty", states the founder of Mares, a company dedicated to accelerating the market entry of Advanced Therapy Medicinal Products on behalf of ATMP innovators. "Consequently, production modalities for therapeutic cells have begun to profit from lessons learned from bioproduction." For example, promising work has been carried out on producing quantities of therapeutic cells in bioreactor systems. These systems are compatible with downstream processing systems such as counterflow centrifugation, all connected through established tubing/aseptic connection systems.

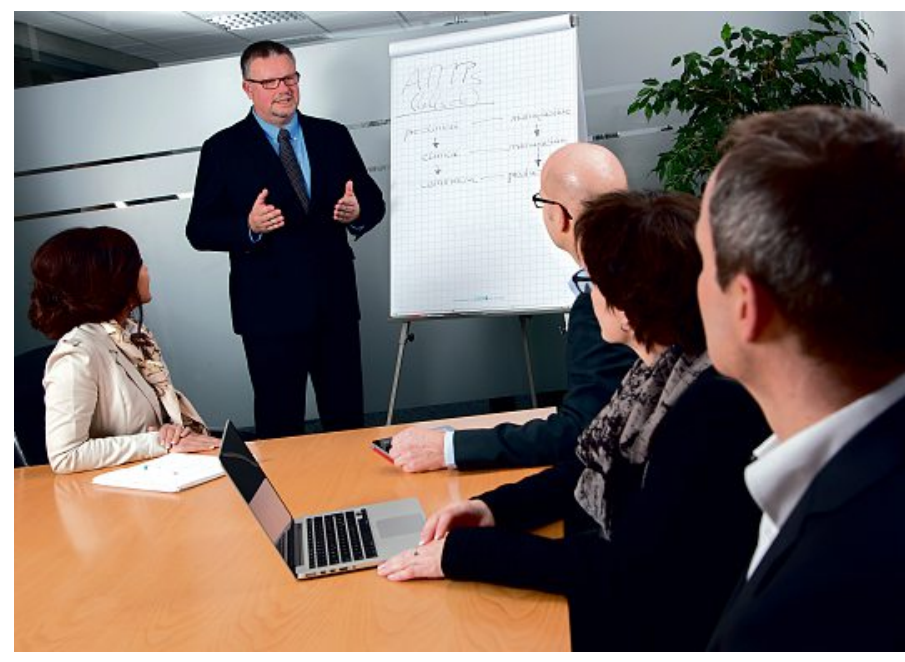

For innovators wishing to commercialize novel therapies, Mares Ltd. provides the knowledge and experience needed to accelerate development, quality, regulatory and manufacturing. For innovators outside the EU, Mares provides legal and regulatory services required by law. (Source: Mares Ltd.)

Another acute headache in the production of therapeutic cells - in terms of both safety and economy - is the use of complex animal products such as bovine serum. "Using bioreactor technology in conjunction with microcarriers is likely to support the generation of processes devoid of such animal products, thus providing for processes that satisfy quantitative and qualitative requirements", says Christian van den Bos, an experienced international R\&D Manager who has been active in the development and scale-up of ATMPs for more than 20 years. "Beyond these immediate concerns we view bioreactors and associated systems as tools to approach physiological conditions during culture expansion of therapeutic cells. We propose this as the central path towards maximizing production efficiency."

www.maresltd.eu

cvandenbos@maresltd.eu

\section{New Concepts in Cellular Immunotherapy}

As regards the implementation of single-use technologies in the development and production of cell and gene therapeutics, Alain Pralong gave us some deeper insights in the application of concepts of $\mathrm{mAb}$ and vaccine development and manufacture to cellular immunotherapy. He has many years of experience as a former Vice President of Product Introduction and Technical Life Cycle Management at GlaxoSmithKline Biologicals and is currently Senior Vice President of Global Manufacturing Operations at Cell Medica in London. The internationally active company develops, manufactures and markets personalized cellular immunotherapeutics for cancer and infectious diseases. According to Alain Pralong, the number of clinical trials conducted in cancer has increased significantly in recent years, with over 5400 studies currently in progress at all stages of product development. This increase has primarily been driven by cellular immunotherapy, which has been made possible by significant progress in the understanding of cancer biology which, in turn, offers a variety of novel therapeutic approaches.

"Autologous cellular immunotherapies currently focus on making the patient's own immune system ready and able to combat the respective cancer", says Alain Pralong. "Either immune cells are specifically selected, activated and expanded before being administered back into the donor patient, as is the case for Cell Medica's CMD-003, or the patient's immune cells are transformed with a chimeric antigen receptor (CAR) to give the patient cancer antigen recognition capabilities after administration that were not present before the treatment, as is the case for leads investigated by Novartis, Kite, Juno and others."

Spectacular clinical results have generated an upsurge in investment in cellular immunotherapy and have massively increased the value of companies involved, given the financial prospects associated with the successful completion of clinical trials and the predicted demand. However, turning these prospects into reality will depend not only on successful clinical results but also on the capability to build a robust and sustainable industry enabling full GMP compliance, supply continuity, and last but not least, accessibility.

https://cellmedica.co.uk/

alain.prolong@cellmedica.co.uk

\section{Smart Technology in Continuous GMP Biomanufacturing}

Dirk Tillich, European Sales Director of the Californiaheadquartered Finesse Solutions, focuses on continuous GMP biomanufacturing. "The current biopharma development and manufacturing models create challenges for the enterprise to meet their goals for high success rates, lower cost and faster time to market", he explains. "With the paradigm shifting from titre to process optimization and analytics, the biopharma business model can be improved using continuous processing to manage complexity. Smart technology exists today which can increase flexibility, decrease costs and improve production." 'Industrie 4.0' describes the Fourth Industrial Revolution and will become an additional success factor for the process industry. Finesse Solutions, with its smart technologies, is well prepared to afford the biopharma industry the benefits of the Industrie 4.0's Modular Automation, defined by organizations such as NAMUR and the 
German ZVEI. Fast and cost-efficient adaptation of production lines to changing processing requirements and volumes, as well as the reduced engineering and validation efforts for new facilities facilitate a shorter time to market and lower cost of goods for traditional and continuous GMP bioprocesses.

www.finesse.com

DTillich@ finesse.com

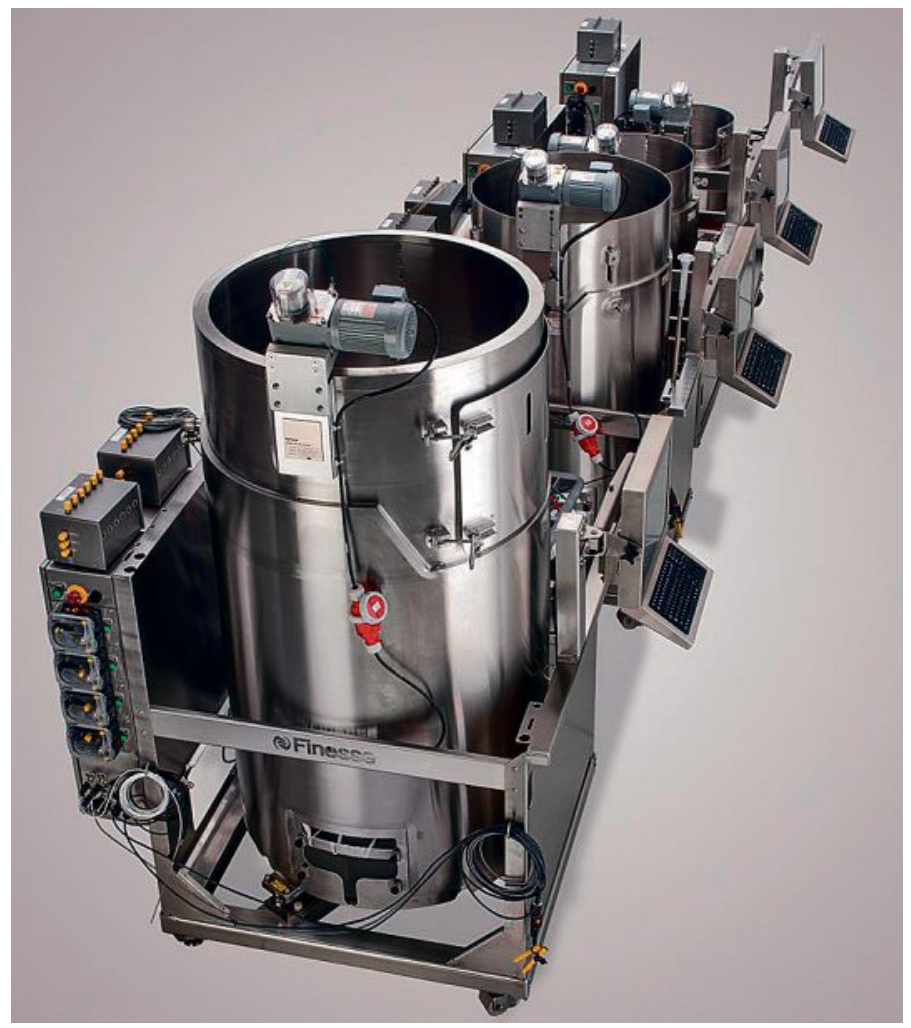

Modular Single Use equipment for flexible GMP production automation at Finesse Solutions Inc. (Source Finesse)

\section{Where Does the Journey Lead?}

In the concluding panel discussion with six internationally renowned experts the focus was on standardization. As in other sectors, in biotechnology we use as much automatization as possible to manufacture a successful product rapidly, in large quantities and at minimal cost. This is in line with standardization. The clients want to be sure of receiving products of a consistently high quality. The manufacturer assures specific standards with its quality management and certifications, while legislation requires compliance with Good Manufacturing Practice (GMP). However, when it comes to the handling of living cells, the implementation of automatization and standardization is much more complex than for products made from inanimate materials. As a result, experts from a wide variety of disciplines have to collaborate and maintain a constant dialogue. This ranges from engineers and technicians specialized in plant construction and mechanical engineering to specialists from natural sciences, and possibly medicine, in order to contribute know-how in the handling of biomaterials and living systems.

But let's be clear about one thing: In today's world we are witnessing a constant and rapid expansion in knowledge. Just think about the domain of functional materials, especially plastics. It will be a long and rocky road to the development of international standards for single-use technologies. But the group headed by Dieter and Regine Eibl at the ZHAW Waedenswil are the ones who are equipped to face the challenge and - together

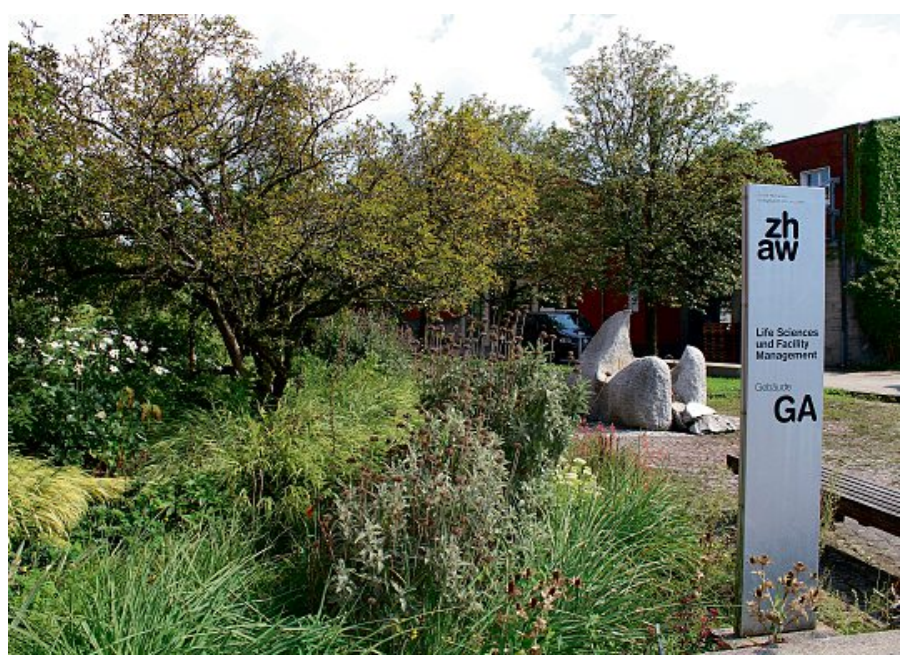

In just a few years, the ZHAW Waedenswil, and especially the group headed by Dieter and Regine Eibl, has become an internationally renowned competence centre for single-use technology and training courses for students and professionals. Close cooperation is maintained with DECHEMA in order to disseminate and share best practice for a broader acceptance of single-use technology. (Source Elsbeth Heinzelmann)

with their global partners - solve problems relating to research and industry in this field.

\section{BioTech 2016 in Retrospect}

"Today, Single-Use Systems (SUS) are an integral component of biopharmaceutical plants. This applies in particular to upstream processing, and from downstream processing through to fill\&finish", concludes Dieter Eibl, Head of Bioprocess Engineering and Cell Cultivation Technique at ZHAW, who set up the event together with his wife Regine Eibl, Head of Cell Cultivation Technique. "For the time being, SUS are dominant in processes involving animal and human cell lines. However, users are hugely interested in implementing SUS more extensively in microbial production systems." The inclusion of SUS has not only changed the processes regarding facility planning and equipment realization, but also opened up new manufacturing plant concepts. Furthermore, producers are putting more and more emphasis on perfusion and concentrated fed batch or continuous operation. The trend is towards flexible and modular manufacturing under the heading of 'Facility of the Future'. "The goal of Biotech 2016 was to present current development trends in the production of bio-, cell- and gene therapeutics. In this way we have again created a perfect platform for networking and the exchange of knowledge."

\section{Prof. Dr. Dieter Eibl}

Head of Bioprocess Engineering and Cell Cultivation Technique Zurich University of Applied Technologies (ZHAW)

E-mail: dieter.eibl@zhaw.ch

Prof. Dr. Regine Eibl

Head of Cell Cultivation Technique

Zurich University of Applied Technologies (ZHAW)

E-mail: regine.eibl@zhaw.ch

\section{www.zhaw.ch/icbt/bioverfahrenstechnik}

